\title{
Risk Stratification of Angioectasia as a Cause of Gastrointestinal Bleeding: Untangling the Spider's Web?
}

\author{
Dejan Micic ${ }^{1} \cdot$ Neil Sengupta $^{1}$
}

Published online: 30 July 2019

(c) Springer Science+Business Media, LLC, part of Springer Nature 2019
Angioectasias (AE) represent one of a variety of vascular gastrointestinal mucosal lesions that can potentially bleed. Initial studies of obscure gastrointestinal bleeding demonstrated an association between calcific aortic stenosis and recurrent melena from bleeding arteriovenous malformations [1]. Although past management included selective angiography [2] and blind right colectomy [3], subsequent advances in flexible endoscopic techniques demonstrated the safety and effectiveness of electrocautery via colonoscopy for management of these lesions [4], thereby highlighting the contribution of the gastroenterologist to the identification and management of bleeding AE.

Due to a lack of understanding of the underlying etiology of vascular intestinal lesions, there until recently has been a lack of a clear consensus definition for an AE. Representing an aberration of the normal vascular structures in the gastrointestinal tract, AE in the small bowel are now defined as "a clearly demarcated, bright-red, flat lesion, consisting of tortuous and clustered capillary dilatations within the mucosal layer (surrounded by intestinal villi) that can range from small (few $\mathrm{mm}$ ) to large (few $\mathrm{cm}$ )" $[5,6]$.

Although similar in appearance, AE are heterogeneous with respect to their presentation (active bleeding, occult bleeding, or asymptomatic) and location within the gastrointestinal tract. AE are most commonly present in the colon ( $0.83 \%$ of healthy asymptomatic adults), most commonly proximal to the hepatic flexure where they often demonstrate a benign natural history with a low rate of bleeding over

Dejan Micic

dmicic@medicine.bsd.uchicago.edu

1 Section of Gastroenterology, Hepatology and Nutrition, Department of Internal Medicine, University of Chicago Medicine, 5841 South Maryland Avenue, MC4076, Chicago, IL 60637, USA
3 years of follow-up [7]. With respect to clinical gastrointestinal bleeding, AE account for 4-7\% of non-variceal upper gastrointestinal bleeding lesions [8] and up to $40 \%$ of colonic bleeding lesions [8]. Among individuals with obscure gastrointestinal bleeding undergoing capsule endoscopy, AE are the most common cause of small bowel bleeding, accounting for $50 \%$ of bleeding lesions [9].

Known clinical conditions associated with the presence of bleeding AE include aortic stenosis [1], von Willebrand disease [10], and chronic kidney disease (CKD) [11]. More recently, AE have also been associated with gastrointestinal bleeding in individuals with left ventricular assist devices (LVADs) with degradation of von Willebrand factor (vWF) [12] as a potential etiology after LVAD implantation. Although a clear pathogenesis has not been described for the formation of AE, numerous hypotheses exist, including intermittent obstruction of submucosal veins with consequent congestion of arterial capillaries and formation of small arteriovenous collaterals, mucosal hypoxemia with resultant neovascularization driven by angiogenesis factors, and deficiency in vWF, having implications for both angiogenesis and hemostasis [8].

Endoscopy remains the primary tool for both diagnosis and therapy, although the understanding of risk factors for the development of symptomatic bleeding from AE remains limited. Consequently, further data are required to help guide which patients may benefit from endoscopic assessment and therapy aimed at mitigating the overall risk of rebleeding from AE [13].

Grooteman et al. contribute a timely systematic review entitled, "A risk assessment of factors for the presence of angiodysplasias during endoscopy and factors contributing to symptomatic bleeding and rebleeds" in this issue of Digestive Diseases and Sciences [14]. The authors performed a systematic review, focusing on (1) risk factors for the presence of AE found during endoscopy, (2) risk factors for AE contributing to gastrointestinal bleeding, and (3) predictors of mortality and rebleeding in individuals with AE. With respect to risk factors for the presence of AE during 
endoscopy, nine individual studies were included, consisting of patients that underwent a variety of endoscopic techniques (including endoscopy, push enteroscopy, and capsule endoscopy, and/or colonoscopy) for suspected gastrointestinal bleeding (overt and obscure). The comparator group underwent endoscopy indicated for bleeding without identification of AE. The primary risk factors for AE included age, CKD, and cardiovascular disease. When studies were pooled that compared patients with suspected gastrointestinal bleeding to a control group of patients undergoing endoscopy without bleeding, added risk factors for detection of AE included the use of proton pump inhibitors, anticoagulants, and a history of venous thromboembolism.

Identification of individuals at high risk of bleeding can be clinically meaningful if endoscopic therapy can be directed at high-risk individuals, thereby limiting repeat hospitalizations or need for additional procedures and therapies for gastrointestinal bleeding. Grooteman et al. identified two studies in which individuals with colonic angioectasias had evidence of blood loss (anemia, positive fecal occult blood, hematochezia, or melena in the endoscopy report [15]) or active bleeding at the time of colonoscopy [16]. Risk factors potentially contributing to bleeding from AE included age $>80$ years and multiple AE identified on colonoscopy. Although further data are required before broad recommendations can be made for treatment of asymptomatic $\mathrm{AE}$ found at the time of colonoscopy for non-bleeding indications, attention should be paid to the right colon, particularly in elderly patients in whom the indication for endoscopy is often for anemia or gastrointestinal bleeding.

Lastly, risk factors for rebleeding attributed to $\mathrm{AE}$ were summarized from six studies (primarily utilizing capsule endoscopy and small bowel enteroscopy) in which rebleeding was associated with cirrhosis, a bleeding locus in the distal small bowel, and multiple AE. Unfortunately, since the contribution of endoscopic treatment toward the achievement of hemostasis was not uniformly collected in these studies, no further data were available regarding the understanding of the contribution of endoscopic cauterization toward the reduction of rebleeding risk [17]. Further studies will be required to quantify the benefit of endoscopic therapies, in particular those delivered to small bowel AE, and reductions in rebleeding risk.

This study provides a clear synthesis of valuable existing data as well as guidance for clinicians that encounter individuals at risk of bleeding from $\mathrm{AE}$ or detect $\mathrm{AE}$ during the course of routine endoscopy. The greatest strength is the large number of included studies and overall participants, but this large sample size also is a primary limitation due to the heterogeneous nature of the included studies and patient populations, which limited the ability to perform a formal meta-analysis for clinically significant risk factors related to development of bleeding and rebleeding. Future clinical studies need to clearly define study aims with respect to $\mathrm{AE}$ location (gastric, colonic, and/or small bowel) and bleeding presentation (overt bleeding with melena or hematochezia or iron deficiency anemia with suspected small bowel bleeding) that will enable meta-analytic techniques to combine data and better define indications for endoscopic assessment as well as management techniques.

In dividing the systematic review into three primary aims, Grooteman et al. attempt to limit the heterogeneity of included studies, but in doing so also limited the number of studies in each aim, thereby increasing the likelihood of bias driven by a single study. With respect to mortality, the largest study included 85,971 individuals [18] or 93\% of included patients within the systematic review, and 99\% of patients in the aim directed at rebleeding and mortality. The included study by Serrao et al. identified individuals with bleeding $\mathrm{AE}$ in a national database by using hospital discharge ICD-9 diagnoses. Although the reported sensitivity of ICD-9 codes for bleeding AE is high, the specificity of $\mathrm{AE}$ as the cause of bleeding has not been determined, therefore limiting the conclusions that can be drawn from a study utilizing a national database [19]. The limitations of the included studies need to be highlighted when performing a systematic review or meta-analysis at the risk of perpetuating inaccurate associations identified in large datasets.

Through their contribution, Grooteman et al. are driving the evidence base and the general knowledge base regarding the contribution of AE toward gastrointestinal bleeding pathogenesis. As noted by the authors, identification of high- and low-risk patient categories is one of the first steps toward defining appropriate endoscopic or medical management techniques with respect to rebleeding risk from $\mathrm{AE}$. As cardiac comorbidities increase in an aging population, and anticoagulant and antiplatelet therapies become more prevalent, bleeding from AE will remain common [20]. Understanding the clinical risk factors related to bleeding and rebleeding is the first step in providing hypotheses related to the pathogenesis of gastrointestinal AE. Further understanding of the etiology and development of clinical risk scores will ultimately enable the development of alternative and safe medical therapies aimed at reducing the burden of disease from gastrointestinal AE.

Author's contribution DM and NS contributed to conception, design, and drafting of the manuscript.

Funding None.

\section{Compliance with Ethical Standards}

Conflict of interest All authors declare that they have no conflict of interest. 


\section{References}

1. Williams RC Jr. Aortic stenosis and unexplained gastrointestinal bleeding. Arch Intern Med. 1961;108:859-863.

2. Nusbaum M, Baum S, Blakemore WS. Clinical experience with the diagnosis and management of gastrointestinal hemorrhage by selective mesenteric catheterization. Ann Surg. 1969;170:506514. https://doi.org/10.1097/00000658-196909010-00018.

3. Case records of the Massachusetts General Hospital. Weekly clinicopathological exercises. Case 49-1965. N Engl J Med. 1965;273:1096-1105. https://doi.org/10.1056/nejm19651111273 2010.

4. Rogers HG, Adler F. Hemangiomas of the cecum. Colonoscopic diagnosis and therapy. Gastroenterology. 1976;71:1079-1082.

5. Brandt LJ. Terminology for vascular lesions of the GI tract. Gastrointest Endosc. 2018;87:1595-1596. https://doi.org/10.1016/j. gie.2018.01.007.

6. Leenhardt R, Li C, Koulaouzidis A, et al. Nomenclature and semantic description of vascular lesions in small bowel capsule endoscopy: an international Delphi consensus statement. Endosc Int Open. 2019;7:E372-E379. https://doi. org/10.1055/a-0761-9742.

7. Foutch PG, Rex DK, Lieberman DA. Prevalence and natural history of colonic angiodysplasia among healthy asymptomatic people. Am J Gastroenterol. 1995;90:564-567.

8. Sami SS, Al-Araji SA, Ragunath K. Review article: gastrointestinal angiodysplasia - pathogenesis, diagnosis and management. Aliment Pharmacol Ther. 2014;39:15-34. https://doi.org/10.1111/ apt.12527.

9. Liao Z, Gao R, Xu C, Li ZS. Indications and detection, completion, and retention rates of small-bowel capsule endoscopy: a systematic review. Gastrointest Endosc. 2010;71:280-286. https ://doi.org/10.1016/j.gie.2009.09.031.

10. Veyradier A, Balian A, Wolf M, et al. Abnormal von Willebrand factor in bleeding angiodysplasias of the digestive tract. Gastroenterology. 2001;120:346-353.

11. Chalasani N, Cotsonis G, Wilcox CM. Upper gastrointestinal bleeding in patients with chronic renal failure: role of vascular ectasia. Am J Gastroenterol. 1996;91:2329-2332.

12. Bartoli CR, Zhang DM, Hennessy-Strahs S, et al. Clinical and In vitro evidence that left ventricular assist device-induced von Willebrand factor degradation alters angiogenesis. Circ Heart
Fail. 2018;11:e004638. https://doi.org/10.1161/circheartfailur e.117.

13. Sakai E, Endo H, Taguri M, et al. Frequency and risk factors for rebleeding events in patients with small bowel angioectasia. BMC Gastroenterol. 2014;14:200. https://doi.org/10.1186/s1287 6-014-0200-3.

14. Grooteman KV, Holleran G, Matheeuwsen M, van Geenen EJM, McNamara D, Drenth JPH. A risk assessment of factors for the presence of angiodysplasias during endoscopy and factors contributing to symptomatic bleeding and rebleeds. Dig Dis Sci. (Epub ahead of print). https://doi.org/10.1007/s10620-019-05683-7.

15. Diggs NG, Holub JL, Lieberman DA, Eisen GM, Strate LL. Factors that contribute to blood loss in patients with colonic angiodysplasia from a population-based study. Clin Gastroenterol Hepatol. 2011;9:415-420. https://doi.org/10.1016/j.cgh.2011.02.003. quiz e49.

16. Nishimura N, Mizuno M, Shimodate Y, et al. Risk factors for active bleeding from colonic angiodysplasia confirmed by colonoscopic observation. Int J Colorectal Dis. 2016;31:1869-1873. https://doi.org/10.1007/s00384-016-2651-1.

17. Kaufman D, Leslie G, Marya N, et al. Small intestinal angioectasia: characterization, risk factors, and rebleeding. J Clin Gastroenterol. 2017;51:720-727. https://doi.org/10.1097/MCG.00000 00000000663.

18. Serrao S, Jackson C, Juma D, Babayan D, Gerson LB. In-hospital weekend outcomes in patients diagnosed with bleeding gastroduodenal angiodysplasia: a population-based study, 2000 to 2011. Gastrointest Endosc. 2016;84(3):416-423. https://doi. org/10.1016/j.gie.2016.02.046.

19. Cooper GS, Chak A, Lloyd LE, Yurchick PJ, Harper DL, Rosenthal GE. The accuracy of diagnosis and procedural codes for patients with upper GI hemorrhage. Gastrointest Endosc. 2000;51:423-426.

20. Kolb JM, Flack KF, Chatterjee-Murphy P, et al. Locations and mucosal lesions responsible for major gastrointestinal bleeding in patients on warfarin or dabigatran. Dig Dis Sci. 2018;63:1878-1889.

Publisher's Note Springer Nature remains neutral with regard to jurisdictional claims in published maps and institutional affiliations. 